\title{
AN INTEGRAL ON A SPACE OF CONTINUOUS FUNCTIONS
}

\section{BERNARD W. LINDGREN ${ }^{1}$}

1. Introduction. In working on the problem of solving integral equations R. H. Cameron and W. T. Martin have investigated and used the Wiener integral, first given by N. Wiener in [5]. ${ }^{2}$ This integral, defined on the space $C$ of continuous functions $x(t)$ on $0 \leqq t \leqq 1$ satisfying $x(0)=0$, has many of the Lebesgue properties, the result of being based on sets of functions termed by Wiener "quasi-intervals" in much the same manner in which the Lebesgue integral is based on intervals of the real line. Its employment, even as a tool in analysis, imposes the restriction (which has its origin in probability) that the functions involved vanish when the argument vanishes. It is to remove this restriction that we define here over $C^{\prime}$, the space of functions $x(t)$ defined and continuous on $0 \leqq t \leqq 1$, an integral resembling the Wiener integral over $C$. The close relationship of the new integral to that of Wiener will be developed and some integration formulas will be obtained.

For definiteness we list the axioms upon which the theory will be based. We consider a space $S$, with certain of its subsets called intervals satisfying the conditions:

(a) the empty set and $S$ itself are intervals,

(b) the intersection of two intervals is an interval,

(c) the complement of an interval is expressible as a finite union of intervals,

(d) associated with each interval $B$ is a non-negative real number (including possibly $\infty$ ) called its measure, $m(B)$, such that if a finite union of disjoint intervals $\sum B_{i}$ is an interval, then

$$
m\left(\sum B_{\mathbf{i}}\right)=\sum m\left(B_{\mathbf{i}}\right),
$$

(e) if an interval $B$ is included in a countable union of intervals $\sum B_{i}$, then $m(B) \leqq \sum m\left(B_{i}\right)$,

(f) there is a countable set of intervals each of finite measure whose union is $S$.

An outer measure is defined as the infimum of the measures of countable unions of disjoint intervals covering the given set. Measur-

Presented to the Society, December 29, 1949; received by the editors January 30 , 1950 and, in revised form, August 3, 1950.

${ }_{1}^{1}$ This paper is part of a thesis written at the University of Minnesota under the direction of Professor R. H. Cameron.

2 Numbers in brackets refer to the bibliography. 
ability is then defined in the usual way according to the Carathéodory criterion.

It is well known that in the space of reals, sets of the type $(a, b]$ with measure $b-a$ satisfy the imposed conditions, and lead to the usual Lebesgue integral; and that in the space $C$, the quasi-intervals of Wiener - sets of the type $B=E\left[y \in C ; a_{i}<y\left(t_{i}\right) \leqq b_{i}, i=1, \cdots, n\right]$ for given $a_{i}, b_{i}$, and $t_{i}$ such that $0<t_{1}<\cdots<t_{n} \leqq 1$-with measure

$$
\begin{aligned}
m_{w}(B)= & {\left[\pi^{n} t_{1}\left(t_{2}-t_{1}\right) \cdots\left(t_{n}-t_{n-1}\right)\right]^{-1 / 2} } \\
& \cdot \int_{a_{n}}^{b_{n}} \cdots \int_{a_{1}}^{b_{1}} \exp \left\{-\frac{u_{1}^{2}}{t_{1}}-\sum_{2}^{n} \frac{\left(u_{i}-u_{i-1}\right)^{2}}{t_{i}-t_{i-1}}\right\} d u_{1} \cdots d u_{n}
\end{aligned}
$$

satisfy these conditions, and lead to the Wiener integral, $\int_{C}^{w} F[y] d_{w} y$.

2. The space $C^{\prime}$. Let the intervals of $C^{\prime}$ be the null set, the whole space, and sets of the type

$$
B=E\left[x \in C^{\prime} ; a_{i}<x\left(t_{i}\right) \leqq b_{i}, i=1, \cdots, n\right]
$$

for given ${ }^{3} a_{i}, b_{i}$, and $t_{i}$ such that $0 \leqq t_{1}<\cdots<t_{n} \leqq 1$. Let the measure of the empty set be 0 , of $C^{\prime}$ be infinite, and

$$
m(B)=\int_{\mathscr{D}} E(u, t) d u,
$$

where

$$
E(u, t)=\left\{\begin{array}{l}
1, \quad \text { if } n=1, \\
{\left[\pi^{n-1}\left(t_{2}-t_{1}\right) \cdots\left(t_{n}-t_{n-1}\right)\right]^{-1 / 2}} \\
\cdot \exp \left\{-\sum_{2}^{n}\left(u_{i}-u_{i-1}\right)^{2}\left(t_{i}-t_{i-1}\right)^{-1}\right\}, \text { if } n \geqq 2,
\end{array}\right.
$$

and where $\mathfrak{B}$ is the set in $n$-space

$$
\mathfrak{B}=E\left[u=\left(u_{1}, \cdots, u_{n}\right) \in E_{n} ; a_{i}<u_{i} \leqq b_{i}, i=1, \cdots, n\right] .
$$

As in the case of the Wiener measure on $C$, it can be verified that if an interval is expressed in two different forms (as it may be, in view of footnote 3 ), the measures calculated according to (2.2) for the different forms are equal. And it is readily shown that with this choice of interval and measure $C^{\prime}$ satisfies (a)-(d) of the preceding section;

${ }^{3}$ Positive and negative infinity are allowed for $a_{i}$ and $b_{i}$, the inequality being made strict in case $b_{i}=\infty$. 
the proof is, in fact, word for word the same as that for $C$. That axiom (f) is satisfied is seen at once from the relations

$$
\begin{gathered}
C^{\prime}=\sum_{-\infty}^{\infty} E[x ; i<x(0) \leqq i+1], \\
m(E[i<x(0) \leqq i+1])=1
\end{gathered}
$$

Showing that axiom (e) is satisfied is perhaps easiest done by using the fact that it is satisfied in the case of $C$. We first obtain an expression for the measure of an interval in terms of Wiener measure.

Definition. Corresponding to a set $S \subset C^{\prime}$ and a real number $\xi$, let $S-\xi=E[y \in C ; y+\xi \in S]$.

Lemma 2.1. If $B$ is an interval in $C^{\prime}, B-\xi$ is for each $\xi$ an interval in $C$; also, $m_{v}(B-\xi)$ is a measurable function of $\xi$, and

$$
m(B)=\int_{-\infty}^{\infty} m_{w}(B-\xi) d \xi .
$$

Proof. Let $B$ be the interval (2.1). Then $B-\xi$ is the interval in $C$

$$
B-\xi=E\left[y \in C ; a_{i}-\xi<y\left(t_{i}\right) \leqq b_{i}-\xi, i=1, \cdots, n\right] .
$$

Assume for the moment that $t_{1}>0$. Then the Wiener measure of $B-\xi$ is

$$
\begin{aligned}
& {\left[\pi^{n} t_{1}\left(t_{2}-t_{1}\right) \cdots\left(t_{n}-t_{n-1}\right)\right]^{-1 / 2}} \\
& \quad \cdot \int_{a_{n}-\xi}^{b_{n}-\xi} \cdots \int_{a_{1}-\xi}^{b_{1}-\xi} \exp \left\{-\frac{u_{1}^{2}}{t_{1}}-\sum_{2}^{n} \frac{\left(u_{i}-u_{i-1}\right)^{2}}{t_{i}-t_{i-1}}\right\} d u_{1} \cdots d u_{n} .
\end{aligned}
$$

Performing the translation $u_{i}=v_{i}-\xi$ for $i=1, \cdots, n$, we have

$$
m_{w}(B-\xi)=\left[\pi t_{1}\right]^{-1 / 2} \int_{\mathfrak{O}} E(v, t) \exp \left\{-\frac{\left(v_{1}-\xi\right)^{2}}{t_{1}}\right\} d v .
$$

The lemma then follows at once for $t_{1}>0$ upon using the Fubini theorem to integrate first on $\xi$.

In the case when $t_{1}=0, B-\xi$ is empty if $\xi$ is not in the range $\left(a_{1}, b_{1}\right]$, and is an $(n-1)$-dimensional interval (or, if $n=1$, then is all of $C$ ) for $\xi$ in that range. Then

$$
\int_{-\infty}^{\infty} m_{w}(B-\xi) d \xi=\int_{a_{1}}^{b_{1}} m_{w}(B-\xi) d \xi .
$$

If in $m_{v}(B-\xi)$ we again translate by means of $u_{i}=v_{i}-\xi$, for $i=2$, 
$\cdots, n$, and let $\xi=v_{1}$, we have

$$
\begin{gathered}
\int_{-\infty}^{\infty} m_{w}(B-\xi) d \xi=\left[\pi^{n-1} t_{2}\left(t_{3}-t_{2}\right) \cdots\left(t_{n}-t_{n-1}\right)\right]^{-1 / 2} \int_{a_{1}}^{b_{1}} \int_{a_{n}}^{b_{n}} \\
\cdots \int_{a_{2}}^{b_{2}} \exp \left\{-\frac{\left(v_{2}-v_{1}\right)^{2}}{t_{2}-t_{1}}-\sum_{3}^{n} \frac{\left(v_{i}-v_{i-1}\right)^{2}}{t_{i}-t_{i-1}}\right\} d v_{2} \cdots d v_{n} d v_{1} \\
=\int_{\mathfrak{B}} E(v, t) d v=m(B),
\end{gathered}
$$

again using the Fubini theorem, and noting that $t_{2}=t_{2}-t_{1}$.

Now suppose that $B$ and the sets of the sequence $\left\{B_{i}\right\}$ are intervals and that $B \in \sum B_{i}$. If $y \in B-\xi$, for some $k y+\xi \in B_{k}$, or $y \in B_{k}-\xi$, so that $y \in \sum\left(B_{k}-\xi\right)$. But then

$$
m(B)=\int_{-\infty}^{\infty} m_{w}(B-\xi) d \xi \leqq \int_{-\infty}^{\infty} \sum m_{w}\left(B_{k}-\xi\right) d \xi=\sum m\left(B_{k}\right),
$$

using Lemma 2.1 and the fact that axiom (e) holds in the case of Wiener measure. We have thus shown that $C^{\prime}$ satisfies axioms (a)-(f); upon it then is based a measure which we shall refer to as $w^{\prime}$-measure, and an integral to be denoted by $\int_{C^{\prime}} F[x] d x$.

A consequence of the definition of measure is the following result.

Theorem 2.1. Let $0 \leqq t_{1}<\cdots<t_{n} \leqq 1$ be fixed. Let $\mathbb{S}$ be a set in $E_{n}$, and let the corresponding letter $S$ denote the set

$$
S=E\left[x \in C^{\prime} ;\left(x\left(t_{1}\right), x\left(t_{2}\right), \cdots, x\left(t_{n}\right)\right) \in \mathfrak{S}\right] .
$$

Then if $\mathfrak{S}$ is a measurable set in $E_{n}$, the set $S$ is w'-measurable, and its measure is $\int_{\varsigma} E(u, t) d u$, where $E(u, t)$ is given by (2.3).

Proof. The theorem is clearly true in the case where $\subseteq$ is an interval in $E_{n}$ (that is, a half open $n$-dimensional rectangular parallelepiped), since then $S$ is an interval in $C^{\prime}$, and $m(S)$ is given by (2.2). Then proceeding from intervals to measurable sets in the usual fashion one obtains the more general result - even in the case where $S$ is not of finite measure, by virtue of axiom (f).

3. Relation to Wiener measure. We now extend (2.5) to measurable sets, and show the relation of $C^{\prime}$ to the product space $C \otimes E_{1}$. The results may be summarized in the following theorems:

Theorem 3.1. $F[x]$ is measurable in $C^{\prime}$ if and only if $F[y+\xi]$ is measurable as a function of $y$ and $\xi$ in $C \otimes E_{1}$.

THEOREM 3.2. If $F[x]$ is measurable in $C^{\prime}$, and if either (i) $F[x]$ is 
summable over $C^{\prime}$, (ii) $F[y+\xi]$ is summable over $C \otimes E_{1}$, or (iii) $F[y+\xi]$ is summable over $C$ for almost all $\xi$ in $E_{1}$, and $\int_{-\infty}^{\infty} d \xi$ $\cdot \int_{C}^{w}|F[y+\xi]| d_{w} y$ exists and is finite, then the relation

$$
\int_{C^{\prime}} F[x] d x=\int_{C \otimes_{E_{1}}} F[y+\xi] d(y, \xi)=\int_{-\infty}^{\infty} d \xi \int_{C}^{w} F[y+\xi] d_{w} y
$$

holds. Furthermore, (i) (ii), and (iii) are equivalent (and may be omitted if $F[x]$ is of constant sign on $C^{\prime}$ ).

Theorem 3.3. If $S$ is a measurable set in $C^{\prime}$, then $S-\xi$ is Wiener measurable for almost all $\xi, m_{v}(S-\xi)$ is a measurable function of $\xi$, and $m(S)=\int_{-\infty}^{\infty} m_{w}(S-\xi) d \xi$.

The remainder of this section comprises the proof of these theorems. We introduce some notation: Corresponding to the set $S \in C^{\prime}$, let

$$
S \dagger=E\left[(y, \xi) \in C \otimes E_{1} ; y+\xi \in S\right],
$$

and corresponding to the set $A \in C \otimes E_{1}$, let $A^{*}$ denote the set

$$
A^{*}=E\left[x \in C^{\prime} ;(x(t)-x(0), x(0)) \in A\right] .
$$

Easily verified consequences are the following:

(a) $(S \dagger)^{*}=S$, and $\left(A^{*}\right) \dagger=A$.

(b) $\left(\sum S_{\alpha}\right) \dagger=\sum S_{\alpha} \dagger$, and $\left(\sum A_{\alpha}\right)^{*}=\sum A_{\alpha}^{*}$; similarly for intersections.

(c) $S_{\alpha} \dagger-S_{\beta} \dagger=\left(S_{\alpha}-S_{\beta}\right) \dagger$, and $A_{\alpha}^{*}-A_{\beta}^{*}=\left(A_{\alpha}-A_{\beta}\right)^{*}$.

(d) $S_{\alpha} \subset S_{\beta}$ implies $S_{\alpha} \dagger \subset S_{\beta} \dagger$, and $A_{\alpha} \subset A_{\beta}$ implies $A_{\alpha}^{*} \subset A_{\beta}^{*}$.

Lemma 3.1. If $B$ is the one-dimensional interval $E\left[x \in C^{\prime} ; 0 \leqq t^{\prime} \leqq 1\right.$, $\left.a<x\left(t^{\prime}\right)\right]$, then $B \dagger$ is measurable in $C \otimes E_{1}$.

Proof. Let

$$
S_{n, k}=E\left[(y, \xi) \in C \otimes E_{1} ; \frac{k-1}{2^{n}}<\xi \leqq \frac{k}{2^{n}}, a-\frac{k-1}{2^{n}}<y\left(t^{\prime}\right)\right],
$$

an interval in the product space, and let $F_{n}=\sum_{\mathbf{k}=-\infty}^{\infty} S_{n, k}$. Since $y\left(t^{\prime}\right)$ $>a-(k-1) / 2^{n}$ and $\xi>(k-1) / 2^{n}$, it follows that $y\left(t^{\prime}\right)>a-\xi$. That is, for each $n$ and $k, S_{n, k}$ is contained in $B \dagger$, and therefore $F_{n} \subset B \dagger$ for all $n$. Further, $F_{n} \subset F_{n+1}$, so that $\lim F_{n}$ exists and is contained in $B \dagger$.

Next let $(y, \xi)$ be a point in $B \dagger$. Let $y\left(t^{\prime}\right)+\xi-a=\delta$, and choose $m$ such that $\delta>2^{-m}$. Let $j$ be the integer for which $\xi \in\left((j-1) / 2^{m}, j / 2^{m}\right]$. Then $y\left(t^{\prime}\right)>a-\xi+2^{-m} \geqq a-j / 2^{m}+1 / 2^{m}=a-(j-1) / 2^{m}$, and so $(y, \xi)$ is in the interval $S_{m, j}$. Therefore $B \dagger=\lim F_{n}$, from which $B \dagger$ is measurable. 
Lemma 3.2. If $B$ is an interval of $C^{\prime}, B \dagger$ is measurable in $C \otimes E_{1}$, and $m(B)=m(B \dagger)$ (where $m(B \dagger)$ is of course the product space measure).

Proof. Let $B$ be the arbitrary interval (2.1). It is expressible in terms of one-dimensional type intervals of the previous lemma:

$$
B=\prod_{1}^{n}\left\{E\left[x \in C^{\prime} ; a_{i}<x\left(t_{i}\right)\right]-E\left[x \in C^{\prime} ; b_{i}<x\left(t_{i}\right)\right]\right\} .
$$

Hence $B \dagger$ is measurable, and so also is $B-\xi$ (in $C$ ), and (2.5) holds. If $\chi_{S}$ denotes the characteristic function of the set $S$, then

$$
m(B \dagger)=\int_{C \otimes_{E_{1}}} \chi_{B} \dagger(y, \xi) d(y, \xi)=\int_{C \otimes_{E_{1}}} \chi_{B-\xi}[y] d(y, \xi)=m(B)
$$

by the Fubini theorem.

Proceeding then from intervals to measurable sets in the usual way, (3.1) is established in the case where $F[x]$ is the characteristic function of a measurable set in $C^{\prime}$, assuming hypothesis (i). For (ii) we need a further result.

LemmA 3.3. If $B$ is the interval $(a, b] \otimes E\left[y \in C ; a_{i}<y\left(t_{i}\right) \leqq b_{i}\right.$, $i=1, \cdots, n]$ in $C \otimes E_{1}$, then $B^{*}$ is w'-measurable, and $m(B)=m\left(B^{*}\right)$.

Proof. It is clear that $B^{*}$ is an $(n+1)$-dimensional set of the type treated in Theorem 2.1:

$$
B^{*}=E\left[x \in C^{\prime} ; a<x(0) \leqq b, a_{i}+x(0)<x\left(t_{i}\right) \leqq b_{i}+x(0), i=1, \cdots, n\right] .
$$

Referring to the notation of that theorem, we see that $\mathfrak{B}^{*}$ is clearly measurable in $E_{n+1}$, and hence $B^{*}$ is $w^{\prime}$-measurable. Equality of the measures follows from the extension of the previous lemma. And as before this equality is immediately extended to measurable sets, and to the characteristic functions thereof.

Theorems 3.1 and 3.2 follow then in the usual way by proceeding from characteristic functions to measurable functions which assume only a finite set of values, to non-negative measurable functions, to arbitrary measurable functions. Theorem 3.3 is corollary to Theorem 3.2. We remark that the converse of Theorem 3.3 is not true, as construction of a counter-example will show.

4. Various properties. In some calculations of integrals using the Fubini theorem in the product space $C^{\prime} \otimes[0,1]$, it is necessary to know the following:

THEOREM 4.1. The functional $F[x \mid t]=x(t)$ is measurable in $C^{\prime}$ $\otimes[0,1]$. 
We omit the proof, since it is identical with the proof of the corresponding result in the case of $C$ with the Wiener measure. Another measurability result is this:

Theorem 4.2. A functional $F[x]$ continuous in the uniform topology is measurable.

Proof. If $x_{0}$ is a fixed point of $C^{\prime}$, the functional

$$
\sup _{\text {binary } t}\left|x(t)-x_{0}(t)\right|=\max _{0 \leqq t \leqq 1}\left|x(t)-x_{0}(t)\right|=\left\|x(t)-x_{0}(t)\right\|
$$

is measurable. But then so also is a uniform neighborhood of $x_{0}$. Now $C^{\prime}$ is a complete metric space with respect to the uniform norm, and is separable in the uniform topology; hence any open set in $C^{\prime}$, being expressible as a countable union of uniform neighborhoods, is measurable. Then if $F[x]$ is continuous, the set of $x$ for which $F[x]<M$ (for any real $M$ ) is the inverse image of the open set $(-\infty, M)$ under a continuous mapping and is hence open, and so measurable. Thus $F[x]$ is measurable.

THEOREM 4.3. If $f(u)$ is Borel measurable in $E_{1}$, and $\zeta(t)$ is of bounded variation on $[0,1]$, then $f\left(\int_{0}^{1} \zeta(t) d x(t)\right)$ is $w^{\prime}$-measurable. ${ }^{4}$

For, since the Stieltjes integral exists, it is expressible as the limit of a sequence of clearly measurable functionals (the approximating sums) and is thus measurable, as is any polynomial in the integral. But $f(u)$ is a Baire function, the result of a sequence of limit operations based on polynomials in $u$. At each step in this sequence of operations measurability is preserved, and the theorem follows by transfinite induction.

Two theorems known to hold in $C$ with the Wiener measure have the following analogues.

THEOREM 4.4. The set $S$ of functions of $C^{\prime}$ zohich do not satisfy for a given $\gamma<1 / 2$ the Hölder condition

$$
|x(t)-x(u)|<h|t-u|^{\gamma}, \text { for all } t \text { and } u \text { in }[0,1] \text {, }
$$

for some real $h$, is of measure zero.

THEOREM 4.5. The set $T$ of functions of $C^{\prime}$ which possess derivatives at at least one point is of measure zero.

Proofs are almost identical, and we give that of the first theorem. Using the notation of the preceding section,

4 Stieltjes integrals are understood to be Riemann-Stieltjes integrals. 


$$
S \dagger=E\left[y \in C, \xi \in E_{1} ; y+\xi \in S\right]=(S \cap C) \otimes E_{1} .
$$

But $S \cap C$ is known ${ }^{5}$ to be a null set in $C$. Hence $S \dagger$ is a null set in $C \otimes E_{1}$, and by Theorem 3.2, $S$ is a null set in $C^{\prime}$.

5. Integration formulas. Extending Theorem 2.1 in the usual way from characteristic functions of measurable sets to measurable functions, one obtains immediately:

THEOREM 5.1. Let $0 \leqq t_{1}<\cdots<t_{n} \leqq 1$ be fixed, and let $G\left(u_{1}, \cdots, u_{n}\right)$ be measurable in $E_{n}$. Then if either $G\left(x\left(t_{1}\right), \cdots, x\left(t_{n}\right)\right)$ is summable over $C^{\prime}$ or $G\left(u_{1}, \cdots, u_{n}\right) E(u, t)$ is summable over $E_{n}$, the other is also summable, and the relation

holds.

$$
\int_{C^{\prime}} G\left(x\left(t_{1}\right), \cdots, x\left(t_{n}\right)\right) d x=\int_{E_{n}} G(u) E(u, t) d u
$$

Using Lemma 3 of [1] for Wiener integrals we obtain a formula similar to the one given there:

THEOREM 5.2. If $\beta(t)$ is of bounded variation on $[0,1], \beta(1)=0$ $\neq \beta(0)$, and if the integrand on the left is measurable and that on the right is of $L_{1}(-\infty, \infty)$, then

$$
\int_{C^{\prime}} f\left(\int_{0}^{1} x(t) d \beta(t)\right) d x=|\beta(0)|^{-1} \int_{-\infty}^{\infty} f(u) d u .
$$

Proof. If $f(u)$ is of $L_{1}$, and $A=\left(\int_{0}^{1} \beta^{2}(t) d t\right)^{1 / 2}$,

$$
\begin{aligned}
\frac{\int_{-\infty}^{\infty} f(\eta) d \eta}{|\beta(0)|} & =\frac{1}{\pi^{1 / 2}} \int_{-\infty}^{\infty} e^{-u^{2}}\left\{\int_{-\infty}^{\infty} f(\eta) \frac{d \eta}{|\beta(1)-\beta(0)|}\right\} d u \\
& =\int_{-\infty}^{\infty} d \xi \frac{1}{\pi^{1 / 2}} \int_{-\infty}^{\infty} e^{-u^{2}} f(A u+\xi[\beta(1)-\beta(0)]) d u \\
& =\int_{-\infty}^{\infty} d \xi \int_{C}^{w} f\left(\int_{0}^{1} y(t) d \beta(t)+\xi[\beta(1)-\beta(0)]\right) d_{w} y \\
& =\int_{-\infty}^{\infty} d \xi \int_{C}^{w} f\left(\int_{0}^{1}[y(t)+\xi] d \beta(t)\right) d_{w} y \\
& =\int_{C^{\prime}} f\left(\int_{0}^{1} x(t) d \beta(t)\right) d x,
\end{aligned}
$$

${ }^{5}$ See $[4$, p. 160] and $[5$, p. 219]. 
where we have used Lemma 3 of [1] to replace the inner integral by a Wiener integral, after first making the change of variable $\eta$ $=\xi[\beta(1)-\beta(0)]+A u$ and then interchanging order of integration.

The Wiener formula for integrating the functional $f\left(\int_{0}^{1} \zeta(t) d y(t)\right)$ (see $\left[1\right.$, p. 393] and $\left[4\right.$, p. 151]) does not carry over to $C^{\prime}$ exactly, but has an analogue if a weighting factor is introduced. Corresponding to a given $\eta(t)$ such that $\eta(1) \neq \eta(0)$ which is defined and of bounded variation on $[0,1]$, let $H_{\eta}[x(\cdot)]=H[x]$ be defined by the relation

$$
H[x]=\frac{|\eta(1)-\eta(0)|}{\pi^{1 / 2}} \exp \left\{-\left(\int_{0}^{1} x(t) d \eta(t)\right)^{2}\right\} .
$$

Then $\int_{C^{\prime}} H[x] d x=\int_{-\infty}^{\infty} d \xi \int_{C}^{w} H[y+\xi] d_{w} y=\int_{C}^{w} d_{w} y \int_{-\infty}^{\infty} H[y+\xi] d \xi=1$.

Theorem 5.3. Let $\eta(t)$ be defined as above. Let $\zeta(t)$ be of bounded variation on $[0,1]$, and let $A=\left(\int_{0}^{1} \zeta^{2}(t) d t\right)^{1 / 2}$. Then if $f(u)$ is measurable in $E_{1}, F[x]=f\left(\int_{0}^{1} \zeta(t) d x(t)\right)$ is measurable in $C^{\prime}$; and a necessary and sufficient condition that $H[x] F[x]$ be summable on $C^{\prime}$ is that $e^{-u^{2}} f(A u)$ be of $L_{1}(-\infty, \infty)$. Moreover, if this condition is satisfied,

$$
\int_{C^{\prime}} H[x] f\left(\int_{0}^{1} \zeta(t) d x(t)\right) d x=\frac{1}{\pi^{1 / 2}} \int_{-\infty}^{\infty} e^{-u^{2}} f(A u) d u .
$$

Proof. If we assume that $F[x]$ is summable in $C,(5.2)$ follows by a simple application of (3.1) and Lemma 3 of [1]:

$$
\begin{aligned}
\int_{C^{\prime}} H[x] F[x] d x & =\int_{-\infty}^{\infty} d \xi \int_{C}^{w} H[y+\xi] F[y+\xi] d_{w} y \\
& =\int_{C}^{w} F[y] \int_{-\infty}^{\infty} H[y+\xi] d \xi d_{w} y=\int_{C}^{w} F[y] d_{w} y .
\end{aligned}
$$

Since the converse of Theorem 3.3 is not true, measurability of $F[x]$ cannot be deduced from the above relations and the measurability of $f(u)$. So to complete the proof, we consider first the special case of (5.2) in which $f(u)=e^{\lambda u}$. The corresponding $F[x]$ is measurable by Theorem 4.3, and since $\exp \left\{-u^{2}+\lambda A u\right\}$ is of $L_{1}$, we have

$$
\begin{aligned}
\int_{C^{\prime}} H[x] \exp \left(\lambda \int_{0}^{1} \zeta(t) d x(t)\right) & d x \\
& =\frac{1}{\pi^{1 / 2}} \int_{-\infty}^{\infty} \exp \left(-u^{2}+\lambda A u\right) d u
\end{aligned}
$$

From this point on the proof proceeds exactly as the proof of the Wiener formula given by Cameron and Martin [1, pp. 394-396], (5.3) 
first being established for complex $\lambda$, and then by a Fourier transform method for more general functions $f(u)$, and then for measurable $f(u)$.

An expression analagous to (5.2) involving a functional on $C^{\prime}$ of the type $f\left(\int_{0}^{1} \alpha_{1}(t) d x(t), \cdots, \int_{0}^{1} \alpha_{n}(t) d x(t)\right)$ for an O.N. set $\left\{\alpha_{j}(t)\right\}$ is obtainable from the known Wiener formula for $C$.

Results of Cameron and Martin which have counterparts in $C^{\prime}$ with $w^{\prime}$-measure are the formulas for transformation of independent variable by translation and by a general linear transformation $[1,3]$. The formulas for $C^{\prime}$ are identical in appearance with those for $C$, and follow from the known results for $C$ and Theorem 3.1, although the details are numerous.

Finally, we mention two more formulas, the first a consequence of Theorem 3.1, and the second of Theorem 3.1 applied to Result 2 of [2]:

$$
\begin{array}{r}
\int_{C^{\prime}} F[x(\cdot)+k] d x=\int_{C^{\prime}} F[x(\cdot)] d x \quad(k \text { constant }), \\
\int_{C^{\prime}} \exp \left(-\eta^{2} \int_{0}^{1} x^{2}(t) d t\right) d x=(\pi / \eta \sinh \eta)^{1 / 2} \quad\left(\operatorname{Re} \eta^{2}>\pi^{2} / 4\right) .
\end{array}
$$

\section{BiBLIOGRAPHY}

1. R. H. Cameron and W. T. Martin, Transformations of Wiener integrals under translations, Ann. of Math. vol. 45 (1944) pp. 386-396.

2. - Wiener measure of Hilbert neighborhoods in the space of real continuous functions, Journal of Mathematics and Physics vol. 23 (1944) pp. 195-209.

3. - Transformations of Wiener integrals under a general class of linear transformations, Trans. Amer. Math. Soc. vol. 58 (1945) pp. 184-219.

4. R. E. A. C. Paley and N. Wiener, Fourier transforms in the complex domain, Amer. Math. Soc. Colloquium Publications, vol. 19, New York, 1934.

5. N. Wiener, Generalized harmonic analysis, Acta Math. vol. 55 (1930) pp. 117258.

UNIVERSITY OF MiNNESOTA 